Universidad de Costa Rica
Facultad de Educación
Instituto de Investigación para el Mejoramiento
de la Educación Costamicense

\title{
CAMINOS RECORRIDOS POR LAS POLÍTICAS EDUCATIVAS DE GÉNERO'
}

\section{Dra. Sandra Araya Umaña*}

Resumen: El estudio de las políticas públicas es revelador de la voluntad del Estado y de sus prioridades de acción. Por ello, para conocer el avance que en materia de género y educación ha experimentado el país, es preciso conocer cuáles son las acciones gubernamentales y las disposiciones concretas que se han desplegado. Este escrito tiene como objetivo hacer una presentación preeliminar de las políticas educativas cuya pretensión es procurar o crear condiciones para la equidad de género en el sistema educativo costarricense; específicamente, en los niveles de primaria y secundaria. Con el propósito de propiciar una mayor discusión y reflexión acerca de la temática, se incorpora el concepto de política pública desde las tres dimensiones que caracterizan su formulación. Esto permite clarificar el "centralismo" que juega la representación social de las mujeres y los hombres en la sociedad. Asimismo, se hace una descripción de las principales instancias vinculadas con la temática; es decir, el Ministerio de Educación Publica y el Instituto Nacional de las Mujeres. Para la elaboración de este escrito, se recurrió a información de fuentes primarias y secundarias.

\begin{abstract}
The study of the public policies reveals the State's will and its priorities of action. For that reason, to know the progress in matters of gender and education that the country has experienced, it is necessary to know which are the actions of the Government and the concrete dispositions that have been established. This writing intends to show a preliminar presentation of the educative policies whose aim is to provide or to create conditions for the equity of gender in the Costa Rican educative system; specifically, in elementary school and in high school. In order to encourage a greater discussion and reflection about the theme, the concept of public policy is included from the perspective of the three dimensions of its makeup. This allows to clarify the central role that the social representation of women and men play within the society. Also, a description is made regarding the main instances tied with the theme; that is to say, the Ministry of Public Education and the National Institute of Women. Information from primary and secondary sources was used for the elaboration of this writing.
\end{abstract}

Descriptores: POLÍTICA PÚBLICA/ POLÍTICA EDUCATIVA/ EQUIDAD DE GÉNERO/ FORMACIÓN DOCENTE/ PUBLIC POLICY/ EDUCATIVE POLICY/ GENDER EQUITY/ TEACHER TRAINING

\section{Presentación}

En la actualidad hay un intenso debate acerca de la necesidad de un Estado Benefactor para América Latina. El tema concita los intereses de quienes defienden su mínima expresión en lo social y en lo económico y, entre quienes, por el contrario, abogan

${ }^{*}$ Doctora en Educación de la Universidad de Costa Rica. Licenciada en Trabajo Social de la Universidad de Costa Rica. Investigadora del Instituto de Investigación para el Mejoramiento de la Educación Costarricense (IIMEC). Trabajadora Social del Ministerio de Educación Pública. Presidenta de la Asociación Herediana para la Prevención y Atención de la Violencia Doméstica (APREVID). Especialista en educación y género.

e-mail: sayarau@cariari.ucr.ac.cr

Artículo recibido: 8 de setiembre, 2003

Aprobado: 24 de noviembre, 2003

\footnotetext{
${ }^{1}$ Avance de investigación del proyecto Políticas públicas y equidad de género: Un análisis desde Costa Rica. Instituto de Investigación para el Mejoramiento de la Educación Costarricense, Universidad de Costa Rica.
} 
por su fortalecimiento. Pese a las polémicas y desacuerdos, hay cuatro aspectos en los que sí es posible consensuar: 1) se ha producido una redefinición del Estado; 2) se ha desarrollado un nuevo paradigma con énfasis en las políticas públicas y la gestión estratégica de esas políticas; 3) existe un acuerdo en otorgar prioridad a la eficiencia y eficacia en la gestión estatal y al mejoramiento de la calidad de relación entre agentes estatales y ciudadanos y 4), el consenso en los puntos anteriores debe manifestarse en un profundo cambio en las estructuras, modalidades de organización y marcos normativos del Estado (Arriagada, 1998).

Desde 1990 las funciones tradicionalmente desempeñadas por el Estado han variado como producto de las nuevas exigencias internacionales en el plano económico social y político. En este nuevo escenario, los planteamientos acerca del desarrollo productivo con equidad abrieron en el ámbito público la discusión sobre la equidad social.

Dentro del enfoque de la equidad social ha sido el discurso de igualdad de oportunidades el que más ha concentrado la atención de la nueva orientación estatal. En esta dirección los asuntos relacionados con el género han discurrido, en el ámbito costarricense en general y en el de la educación en particular, con el postulado de igualdad de oportunidades traducido en igualdad de acceso.

Innegable es que la inclusión del tema de género en la agenda estatal ha tenido dos fuertes instigadores: el apoyo de las agencias internacionales y el movimiento feminista. Si bien en el ámbito estatal éste último no tiene la fuerza de las primeras, sí la ha manifestado desde la sociedad civil por medio de la denuncia y la promoción de los derechos de las mujeres.

Sin embargo, aunque asistimos a la creación de condiciones favorables para la redefinición de las funciones en la gestión pública, existen nudos importantes que limitan la inclusión del género. Como veremos, éstos tienen relación con la multiplicidad de actores sociales y políticos involucrados, la complejidad institucional y en especial con las resistencias ideológicas.

\section{Acerca de las Políticas públicas}

Las políticas públicas refieren a un proceso continuo de toma de decisiones del Estado para la resolución de problemas de diferente naturaleza. No son acciones neutrales desprovistas de contenido ideológico ni son el resultado de decisiones unilaterales. Por el contrario, constituyen una forma particular de gestión del Estado para enfrentar los retos del desarrollo social y de la reproducción ideológica del orden social. En este sentido, las 
políticas públicas expresan la "lógica social y cultural" que el Estado debe preservar con el objetivo de garantizar la cohesión y el "orden" del sistema social.

Tres dimensiones caracterizan la formulación de las políticas públicas (Azevedo cfr. Schneckenberg, 2003), a saber:

- Dimensión cognitiva: Relaciona las propuestas innovadoras tanto con el conocimiento técnico-científico, como con las representaciones sociales de los "hacedores de la política" (es decir, con la lectura específica que hacen de la realidad social los dirigentes de un sector, en determinado momento).

- Dimensión instrumental: Implica la búsqueda de metodologías que permitan atacar las causas de los problemas y que, a la vez, articula los datos técnicos con los valores políticos.

- Dimensión normativa. Muestra la relación entre las políticas, los valores y las prácticas culturales y sociales dominantes; articula las políticas al proyecto global que está en curso en la sociedad (así, garantiza que las soluciones pensadas para los problemas, impliquen el respeto y la preservación de los valores vigentes en las relaciones sociales y que se actualizan en las prácticas cotidianas de los individuos y grupos).

De esta manera, las representaciones sociales (RS) ${ }^{2}$ imperantes acerca de las relaciones entre mujeres y hombres; del ideal femenino; de la división sexual del trabajo; de la sexualidad y de los derechos económicos de las mujeres, entre otras, van a estar presentes - a veces de forma sutil y otras no tanto- en las políticas que se formulen para la equidad entre los sexos.

Pero no solo en el plano de la formulación intervienen la Rs. También lo hacen en el de la ejecución pues, aunque se formulen políticas basadas en discursos de género, siempre existirá un desfase entre el nivel discursivo y los resultados que efectivamente se logran alcanzar. $Y$ ello es así debido a las interpretaciones - o distorsiones - de las formulaciones globales, provenientes de las RS de las personas que concurren en el sistema institucional como de la sociedad civil en general.

En síntesis, las formulaciones originales de por sí contienen elementos que responden a lo establecido en el orden social y que están presentes en la dimensiones cognitiva,

\footnotetext{
2 Las RS constituyen sistemas cognitivos en los que es posible reconocer la presencia de estereotipos, opiniones, creencias, valores y normas que suelen tener una orientación actitudinal positiva o negativa. Se constituyen, a su vez, como sistemas de códigos, valores, lógicas clasificatorias, principios interpretativos y orientadores de las prácticas, que definen la llamada conciencia colectiva, la cual se rige con fuerza normativa en tanto instituye los límites y las posibilidades de la forma en que las mujeres y los hombres actúan en el mundo (Araya, s.f).
} 
instrumental y normativa de las políticas pero, también en su ejecución, dichas políticas son transformadas producto de la acción de los agentes sociales que intervienen en ellas.

\subsection{La política educativa como política pública}

La política educativa forma parte de un proyecto social amplio; por ende, debe pensarse en una articulación íntima dialéctica con la planificación global que la sociedad construye como su proyecto, y que se realiza por medio de la acción del Estado (Schneckenberg, 2003). En ella subyace una intencionalidad de cambio o de innovación y en este sentido las reformas educativas se presentan como elementos fundamentales en la elaboración de las propuestas.

Dos aspectos caracterizan la política educativa:

1. Actúa sobre la educación pero no tiene el dominio absoluto sobre ella pues, como práctica social, la educación excede a la escuela y, en consecuencia, incide en ella, ya que su implementación depende de las relaciones establecidas en la práctica escolar.

2. Proyecta la formación de las personas que la sociedad desea perfilar. Sus objetivos son los que definirán qué tipo de individuo y de sociedad se está buscando. Canaliza la educación para fines determinados; “...está cargada de intenciones, y son justamente las intenciones lo que tienen en común todos los tipos de política educativa" (Schneckenberg, 2003, p. 4).

La equidad de género promocionada desde la política educativa costarricense promueve la igualdad de oportunidades entre los sexos por medio, fundamentalmente, del análisis de los estereotipos sexuales en los libros de texto; del estudio y superación de las diferencias que se dan en las actitudes; de los comportamientos y lenguaje de los docentes y las docentes hacia niños y niñas en el aula y de la promoción y potenciación de las mujeres para su ingreso y permanencia en carreras consideradas masculinas. Sin embargo, como veremos en páginas posteriores, dicha igualdad no se ha plasmado en modificaciones sustantivas en el sistema educativo.

En efecto, estas acciones que son inherentes al feminismo liberal -de fuerte influencia en América Latina (Araya, 2003a) - no pretenden cambiar la base ni la estructura de las relaciones de poder que caracterizan las relaciones entre mujeres y hombres. De ahí, que hay grandes temas ausentes en la formulación de las políticas educativas para la 
equidad de género (las nuevas configuraciones familiares, la división sexual del trabajo y lo derechos reproductivos de las mujeres, entre otros).

Dos factores obstaculizan además dicha formulación: 1) Las relaciones de género están cruzadas por dimensiones culturales, políticas e ideológicas que aluden a los aspectos subjetivos de las personas y por tanto, en algunos casos, su sola mención genera una significativa movilización personal; 2) Debido al carácter subjetivo que los atraviesa, los temas de género son definidos dentro del "ámbito privado" y en esa medida parecen y son analizados como si fueran preocupaciones de sentido común y, por tanto, no susceptibles del diseño de políticas públicas (Arriagada,1998, p. 6).

\subsection{1 ¿Quiénes elaboran las políticas educativas?}

Aspectos medulares de las políticas educativas como las cuestiones relativas a qué contenidos enseñar, a quiénes y cómo enseñarlos se encuentran insertos en una estructura de poder, que tradicionalmente está representado por el Estado, el que a su vez delega en un ministerio o secretaría su potestad.

Lo anterior se traduce en que es un grupo pequeño, definido como el portador de habilidades específicas para administrar —aunque no necesariamente sea poseedor de las mismas - el que se encarga de la formulación de las políticas educativas. Es decir, la característica o tónica principal de las políticas o reformas educativas es que son formuladas por grupos restrictivos.

La política educativa debe ser diseñada por un equipo interdisciplinario y transdisciplinario que tenga una visión holística, global de lo que debe hacerse en el país. No puede diseñarse en un cuarto sin ventanas, frente a una computadora. Tiene que ser un equipo que tenga la visión de una política de largo plazo desde la percepción del Estado y nunca de gestión gubernamental (Paniagua, 2002, p.13).

Si bien diferentes investigaciones han señalado que, en buena parte, el fracaso de las políticas y las reformas educativas deviene de este carácter restrictivo (Carr, 1996) lo cierto es que en Costa Rica esta práctica no ha sido desterrada. Las reformas han sido impulsadas desde la cúpula magisterial y aunque se señala (MEP, 1981) que han tenido una participación democrática nacional en cuanto a su ejecución, también se ha reconocido que no es suficiente que al magisterio se le informe si no existe una activa participación desde la génesis misma de las reformas. 
Pareciera ser del más valor estratégico que, el personal docente y en general todas las autoridades educativas, tengan la capacitación necesaria a fin de que sea comprendida una actitud de participación dinámica en los procesos de cambio en la educación costarricense (MEP; 1981, p. 29).

Por otro lado, al estar la política educativa en relación con la estructura de poder, influencia y es influenciada por diversas fuerzas de acción política. Por ello, las políticas educativas pueden surgir también de la imposición de un pequeño grupo o institución social que ejerce el poder sobre la colectividad. La Iglesia Católica es, en este sentido, una de las instituciones que más se ha involucrado en la (re) definición de políticas educativas, particularmente en lo que respecta a la educación para la sexualidad en las escuelas y colegios.

La comunidad educativa internacional y los organismos financieros internacionales son también dos actores cuya acción no se puede obviar en la formulación de las políticas educativas.

En efecto, los foros internacionales contemplan compromisos que de hacerse efectivos cambiarían notablemente las condiciones en la educación y el género en muchos países. Pero debido al hecho que tales compromisos deben ser rendidos en detalle y financiados por cada país en particular, surgen grandes distancias entre las promesas hechas en los foros y lo que luego se traduce a nivel nacional mediante la promulgación de leyes y políticas y acciones concretas (Stromquist, 2003).

Por su parte, la inversión en la educación pública está afectada por las recomendaciones e imperativos de agencias financieras internacionales, en particular el Banco Mundial y el Fondo Monetario Internacional, cuya principal función radica en el pago de la deuda externa por parte de los países en desarrollo. Ello hace que la inversión en servicios educativos y de salud se salgan de las fuerzas políticas internas y pasen a ser asuntos intocables en manos de los ministerios de economía de los países deudores.

Los técnicos en estos ministerios operan con órdenes de mantener el presupuesto anunciado de modo fijo, de no incurrir en déficit y de pagar el servicio de la deuda de modo ininterrumpido. Ya que estas condiciones imperan para la obtención de futuros préstamos, de hecho significa que las decisiones educativas están ya no en el poder ejecutivo o legislativo pero sí en la banca internacional (Stromquist, 2003, p.7). 


\section{Marco normativo nacional para la equidad de género}

El Estado Costarricense ha asumido distintos compromisos internacionales para la promoción de la igualdad de oportunidades y en este sentido ha tomado medidas que incluyen la revisión y la promulgación de leyes nacionales.

La Constitución Política establece que ninguna persona podrá ser sometida a discriminación alguna contraria a la dignidad humana (artículo 33, reformado en 1968) y que nadie será sometido a tratamientos crueles ni degradantes (artículo 40). Establece además, que el Estado procurará el mayor bienestar a todos los habitantes del país (artículo 50), particularmente a las mujeres madres y a sus hijos e hijas (artículos 51 y 55), especialmente en lo que respecta al trabajo y la educación.

En consonancia con los nuevos requerimientos del lenguaje no sexista, dos artículos de la Constitución Política fueron reformados: el artículo 20 que rezaba: "Todo hombre es libre en la República...", y ahora dice: “Toda persona es libre en la República...”, y el artículo 33 que decía "Todo hombre es igual ante la ley y no podrá hacerse discriminación alguna contraria a la dignidad humana", ahora dice "Toda persona es igual ante la ley..."

El papel del Estado costarricense en lo referente a la protección de los derechos de las mujeres se fortalece además con la promulgación de varias leyes en la década de los noventa: la ley de creación de la Delegación de la Mujer (1990), la de Promoción de la Igualdad Social de la Mujer (1990), la de la Defensoría de la Mujer en la Defensoría de los Habitantes (1993), la Ley contra el Hostigamiento Sexual en el Empleo y la Docencia (1995), la Ley contra la Violencia Doméstica (1996), el Código de la Niñez y la Adolescencia (1997), la Ley General de Protección a la Madre Adolescente (1997), el Reglamento sobre Salud Sexual y Reproductiva (1999) y la Ley de Paternidad Responsable (2001).

No obstante, y sin desmerecer los grandes avances en el campo jurídico, en materia de obstáculos materiales, sociales y políticos que limitan la igualdad de las mujeres, el país ha avanzado lentamente debido a la ausencia de políticas públicas tendientes a la transformación cultural que se requiere para la plena igualdad de las mujeres y los hombres.

En efecto, las acciones desplegadas desde la creación de la Oficina de Programas para la Mujer y la Familia en el Ministerio de Cultura, Juventud y Deportes en 1974, hasta las desarrolladas por el Centro de Mujer y Familia (CMF) (institución en la que se transformó la primera) no se tradujeron en acciones de impacto. 
...Su trabajo se había caracterizado durante mucho tiempo, por el desarrollo de acciones puntuales, focalizadas, desvinculadas entre sí y con un carácter meramente asistencial dirigido fundamentalmente a la población femenina ubicada en los cantones de mayor deterioro económico (Centro de Mujer y Familia, 1998, p.19).

La aplicación gubernamental de la Plataforma de Acción de Beijing (que se inició en el período gubernamental 1994-1998) así como una redefinición de las políticas del CMF permitieron en 1996, el diseño y la ejecución de tres planes nacionales: el Plan para la Igualdad de Oportunidades entre Mujeres y Hombres (PIOMH), el Plan Nacional para a Prevención y la Atención de la Violencia Intrafamiliar (PLANOVI) y el Programa de Promoción de la Ciudadanía Activa de las Mujeres, PROCAM (Ramellini, 2000).

A pesar de que el PIOMH pretendió ser un instrumento de políticas públicas que reuniera las actuaciones de la administración pública con el objetivo de lograr la equidad de género en diferentes ámbitos (legislativo, familiar, educativo, de salud, cultural y de comunicación, laboral, ambiental y de toma de decisiones), ello no fue posible por carecer de acciones estratégicas.

Después de una evaluación nos percatamos que el PIOMH no tenía medidas de carácter estratégico. Por ejemplo, se hablaba de módulos de capacitación, pero no se decía que era lo que se pretendía con los módulos. El módulo es el medio pero no se indicaba si se pretendía un cambio de actitud; que existiera un cambio institucional en determinadas áreas, etc. O sea, había muchas acciones que no eran el centro de interés y no eran de impacto (Araya, 2003b).

A inicios de 1998, el Centro Nacional para el Desarrollo de la Mujer y la Familia se transformó en el Instituto Nacional de las Mujeres (INAMU). Además, en mayo de 1998, a raíz de las elecciones presidenciales se designó por primera vez a una Ministra de la Condición de la Mujer, quien se desempeña a su vez como Directora Ejecutiva del INAMU.

Los fines del INAMU son (Ley de Creación, 1998):

1. Formular e impulsar la política nacional para la igualdad y equidad de género, en coordinación con las instituciones públicas, las instancias estatales que desarrollan programas para las mujeres y las organizaciones sociales.

2. Proteger los derechos de las mujeres consagrados tanto en declaraciones, convenciones y tratados internacionales como en el ordenamiento jurídico costarricense; promover la igualdad entre los géneros y propiciar acciones tendientes a mejorar la situación de las mujeres. 
3. Coordinar y vigilar que las instituciones públicas establezcan y ejecuten las políticas nacionales, sociales y de desarrollo humano, así como las acciones sectoriales e institucionales de la política nacional para la igualdad y equidad de género.

4. Propiciar la participación social, política, cultural y económica de las mujeres y el pleno goce de sus derechos humanos, en condiciones de igualdad y equidad con los hombres (Artículo 3).

En la actualidad, el INAMU se ha planteado dentro de sus desafíos institucionales "Diseñar y poner en operación el Sistema Nacional para la lgualdad y la Equidad de Género, en el que se inscriba la política nacional' (INAMU, 2002, p. 6). Debido a que este plan es para el período 2002-2006 debemos esperar los frutos de estas iniciativas.

\section{Equidad de género y educación}

La creación, en 1974, de la Oficina de Programas para la Mujer y la Familia en el Ministerio de Cultura, Juventud y Deportes como la instancia encargada de coordinar las acciones relacionadas con la celebración del Año Internacional de la Mujer en 1975, es un claro indicador de que el Estado costarricense ha mostrado preocupación por la temática relacionada con las mujeres. $\mathrm{Y}$ aunque las acciones han sido puntuales y con carencias presupuestarias, este hecho marca una gran diferencia con otros países latinoamericanos (Brasil y Perú, por ejemplo) en los que la acción estatal se ha caracterizado por ser más lenta y tímida.

En materia de género y educación la primera acción realizada data de 1982 y consistió en la firma de un Convenio de Cooperación Interinstitucional entre el MEP y el Ministerio de Cultura, Juventud y Deportes, al cual estaba adscrita la Dirección General de Programas para la Mujer y Familia. Con dicho convenio se pretendía promover la actualización de programas de educación y textos escolares, de manera que no se establecieran diferentes patrones de conducta para cada sexo ${ }^{3}$.

Para el cumplimiento de lo anterior se creó una comisión interinstitucional cuyo objetivo era la revisión de los textos educativos de la "Serie Hacia la Luz". Sin embargo, la proyección del cambio de los roles provocaba reticencia en los mandos medios involucrados, lo cual produjo resultados muy tímidos.

3 Según entrevista personal (Araya 2003b) lo que se pretendía era abordar la discriminación y marginación de las mujeres pues en aquel momento no se hablaba de género. 
En 1984 el estado costarricense ratifica la Convención sobre la Eliminación de Todas las Formas de Discriminación contra la Mujer que, en su artículo 10, establece la eliminación de todo concepto estereotipado de los papeles masculinos y femeninos en todos los niveles y en todas las formas de enseñanza. Sin embargo, dicha ratificación —con excepción de las acciones desarrolladas por el convenio mencionado- no genera acciones específicas, sino hasta 1991 cuando el CMF y el MEP reinician coordinaciones por medio del espacio que abrió el Proyecto Subregional "Mujeres, Género y Desarrollo del Fondo de las Naciones Unidas para el Desarrollo de la Infancia (UNIFEM) y cuya preocupación central era la producción de material didáctico libre de estereotipos de género.

Este proyecto estaba en consonancia con la producción de investigaciones acerca de los libros de texto que en nuestro país - y en América Latina, en general- se empezó a gestar a finales de la década de los 80 e inicios de los noventa. Gran parte de esta producción (González, 1987, 1990; Montes de Oca y Rosales, 1993; UNICEF/UNIFEM, 1989, 1991) se inspiraron en la investigación pionera de Andree Michel (1987) y se basaron en la metodología de análisis de frecuencia de imágenes estereotipadas. Concluyeron, en términos generales, que la invisibilidad femenina es la nota característica de los libros de texto y cuentos infantiles y si se le visibiliza se hace en papeles de cuido y servicio (Araya, 2001a). Asimismo, la vasta producción en este campo permitió delinear diferentes propuestas cuyo objetivo era abordar el sexismo en la educación primaria (UNICEF/UNIFEM, 1990; UNESCO 1992; Montes de Oca 1994; Paredes 1994; Paredes et al, 1994; Centro Nacional para el Desarrollo de la Mujer y la Familia1997 a, b, c; Ibarra 1997). Estas propuestas coincidían en la necesidad de revisar y cambiar los códigos y formas del lenguaje; evitar el uso de genéricos con la consecuente utilización del femenino y el masculino y, en general, señalaban como tarea inmediata de la educación, la revisión y superación de todas las formas sexistas lingüísticas.

Como producto de lo anterior, en el país el texto escolar Paco y Lola fue eliminado y otros tuvieron algunas modificaciones en las imágenes, no obstante, hubo serias limitaciones para impulsar desde el MEP un lenguaje no sexista y erradicar las imágenes estereotipadas de género en los libros debido a la multiplicidad de actores sociales que intervienen en el proceso educativo (por ejemplo, las modificaciones en las imágenes que inicialmente se lograban concretar, se revertían en la edición final pues eran nuevamente cambiadas a su aspecto tradicional por quienes se encargaban de esta última tarea).

Por otra parte, en el plano de la normativa en 1990 se promulga la Ley de Promoción de la Igualdad Social de la Mujer, que en su capítulo V, artículo 17, prohíbe que en cualquier Volumen 3, Número 2, Año 200310 
institución educativa nacional, se utilicen contenidos, métodos e instrumentos pedagógicos, que asignen papeles para hombres y mujeres que sean contrarios a la igualdad social y la complementariedad de los géneros o que mantengan una condición subalterna de la mujer. Dos años después surge en el MEP el "Proyecto Sistema de Mejoramiento de la Calidad de la Educación en I y II Ciclo para el Fomento de la Excelencia y la Equidad (SIMED), cuyo objetivo fundamental era ampliar, consolidar y profundizar la integración de los ejes formativos, especialmente en educación ambiental y la condición de género en el currículo a nivel institucional y de aula (Fernández, 2001).

La creación del SIMED y la promulgación de la Ley de Promoción de la Igualdad Social de la Mujer, sin embargo, no significaron el despegue de proyectos en esta dirección, sino hasta 1995 cuando el SIMED coordina con la Oficina Ministerial de la Mujer del MEP (OMM/MEP).

La OMM/MEP, al igual que el resto de Oficinas Ministeriales y Sectoriales de la Mujer se creó por acuerdo firme del Consejo de Gobierno el 12 de mayo de 1994 con el propósito de que velara por el cumplimiento de las políticas gubernamentales para la equidad de género. Fue la primera en crearse en el sector público y en el plano de las formulaciones no así en la práctica - era la responsable de la ejecución del PIOMH en el sector educativo cuyos objetivos eran:

a Garantizar el principio de igualdad de oportunidades entre hombres y mujeres en todas las dimensiones y niveles del sistema educativo y de formación profesional.

a Utilizar los materiales didácticos como instrumento para trasmitir y reforzar la igualdad de oportunidades entre mujeres y hombres.

a Promover la modificación de las prácticas sexistas que se presentan en la interacción cotidiana entre educadores y educandos (as).

- Promover la participación igualitaria de mujeres y hombres en los puestos administrativos y de toma de decisión del sistema educativo.

- Promover las condiciones necesarias para que las adolescentes o madres puedan continuar con sus estudios.

- Alentar la participación de las mujeres en la práctica de los deportes.

- Fortalecer los programas formativos para las mujeres adultas desarrollados por el Ministerio de Educación Pública de manera que se adecuen a sus intereses.

a Promover en el nivel universitario estudios, investigaciones y acciones educativas relacionados con la igualdad de oportunidades entre los sexos y favorecer la difusión de los mismos. 
- Incorporar en el proceso educativo las necesidades, aspiraciones y prácticas culturales de los diferentes grupos que conforman la identidad pluricultural y multilingüe de la población costarricense (Centro Nacional para el Desarrollo de la Mujer y la Familia, 1996, p. 26-31).

Espacialmente, en un inicio la OMM/MEP se ubicó en el Despacho del Viceministerio y no pudo cumplir con su principal responsabilidad, cual fue "velar por la incorporación del enfoque de género en la documentación y actividades del ministerio" debido a una serie de tropiezos, entre ellos, la falta de presupuesto y el perfil de las encargadas.

Por estas razones, la coordinación entre el SIMED y la OMM/MEP no impactó en el sistema educativo pues las funciones de la segunda se traducían en conmemoración de fechas significativas ( 8 de marzo, 25 de noviembre); elaboración de afiches y sesiones de capacitación a directores y directoras regionales que no lograron trascender de este nivel.

\subsection{Política Educativa hacia el Siglo XXI}

Fue aprobada por el Consejo Superior de Educación en noviembre de 1994 y de ella se derivó el EDU 2005 con el fin de traducirla en dispositivos de acción. Desde entonces, es la que rige el país pues aunque en las administraciones subsiguientes se han elaborado planes de acción y planes estratégicos (con diferentes énfasis según sea la prioridad gubernamental), en nueve años no ha existido ningún esfuerzo por revisar o formular nuevas políticas educativas.

EI EDU 2005 plantea, entre otros, el siguiente desafío: "Eliminar del sistema en general y de la educación en particular, elementos que generen desigualdades basadas en las diferencias de género o de cualquier otra naturaleza"(Chavarría, 1998, p. 5).

El problema educativo suele asociarse a un problema de acceso y de calidad y lo relacionado con el género es visualizado como un problema distractor de su solución. De ahí que, a pesar del anterior reconocimiento, en el EDU 2005 el tema de género está ausente y se define como problemas prioritarios: (a) equipo, mobiliarios, infraestructura; b) universalización de la cobertura informática; $c$ ) la formación y la capacitación docente, $d$ ) la modernización tecnológica, gerencial y de calidad de los servicios administrativos que brinda el MEP y e) cobertura de la educación (Chavarría, 1998, 159).

Desde el EDU 2005 la finalidad prioritaria de la OMM/MEP son los materiales didácticos como instrumentos para trasmitir y reforzar la igualdad de oportunidades desde la niñez, con lo cual se "regresa", en buena medida, a la preocupación por los materiales didácticos, característica de la década del 80 e inicios del 90. 


\subsection{Construcción de una Cultura Equitativa de Género en la Escuela Líder ${ }^{4}$.}

En 1998, el INAMU y el SIMED — que para el desarrollo de sus programas contaba con el apoyo económico de los Países Bajos - impulsaron en 20 escuelas líderes el Subproyecto Construcción de una Cultura Equitativa de Género en la Escuela Líder.

El sustento conceptual de ste proyecto contiene elementos interesantes de destacar por cuanto evidencian un fuerte desarrollo teórico (Fernández, 2001).

- Se reconoce que la paridad numérica en la matrícula escolar y universitaria no es sinónima de paridad genérica.

- Se señala la feminización de algunas carreras (en las áreas de las ciencias sociales y de letras), y su subsecuente desvalorización en el mercado laboral.

- Se señala el currículo oculto como uno de los instrumentos más sutiles para establecer distinciones en el tratamiento escolar de las personas de acuerdo con el sexo.

- Se reconoce que la educación es androcéntrica ya que considera como hegemónica la visión y las experiencias masculinas al identificarlas con lo humano, ignorando la perspectiva femenina.

- Se indica que la estructura organizativa de los centros educativos es otro de los componentes que incide en los valores y normas que el estudiantado interioriza pues aunque la mayoría de docentes en la primaria son mujeres no existe un correlato en el número que ocupan puestos de dirección.

Asimismo y tomando como referencia diferentes autoras, se señalan las definiciones de sistemas de género, equidad y género que revelan un considerable desarrollo teórico.

4 Escuela Líder: Es la propuesta de un cambio de paradigma escolar, de concepción y de acción , tanto en los procesos de gestión, como en los técnicos pedagógicos, de tal manera que al tiempo que se va construyendo el proyecto educativo del centro escolar, se vaya generando una nueva cultura de desarrollo humano y de aprendizaje en y de la institución (Fernández, 2001, p. 4). Al concebir a las Escuelas Líderes se pensó que dichas escuelas además de generar su propio cambio, generarían también capacidad para influir cambios en otras escuelas tradicionales o de avanzada de su circuito y esto es más exactamente lo que entraría a definir su liderazgo. Es en este sentido que el Proyecto de Apoyo al SIMED estableció en el marco de su tercera fase que las Escuelas Líderes ejerzan influencia o liderazgo sobre otras 600 escuelas del país para que todas busquen y encuentren su mejoramiento y el mejoramiento de la calidad de la educación. A estas 600 escuelas se les ha bautizado con el nombre genérico de escuelas de Proyección, entre las cuales están comprendidas 78 escuelas de Atención Prioritaria y 84 unidocentes (MEP, 1998). 
No obstante, y a pesar de la estrategia operativa de conformar equipos en diferentes niveles, los planteamientos teóricos y las intencionalidades no se tradujeron en aspectos concretos de acción, debido, entre otras, a las siguientes limitantes (MEP, 2002) ${ }^{5}$ :

u Rotación del personal: Parte del personal docente que recibió la primera capacitación se trasladó a otros centros educativos.

a Uso inadecuado del material: Algunas escuelas se quedaron sin el material didáctico pues hubo quienes lo consideraron de uso personal y no se dejó en la biblioteca o bien en algunas escuelas, a pesar de que estaba, no se usaba.

a Centralismo: Ausencia de participación del estudiantado y de las familias en las distintas etapas de ejecución del subproyecto.

- Aspectos administrativos: En algunos casos, solo hubo apoyo del director o directora, pues los supervisores y asesores no se involucraron por tener exceso de trabajo administrativo.

En la actualidad la continuidad del proyecto está siendo evaluada pues se ignora si se va a trabajar con las mismas escuelas líderes debido a las nuevas exigencias del Plan Vida Nueva ${ }^{6}$ y además por el desinterés de algunos docentes que participaron en la experiencia pasada (Araya, 2003c).

\subsection{Educación para la Sexualidad}

En el campo de la educación para la sexualidad, Costa Rica ha elaborado diferentes legislaciones, documentos y propuestas. De esta manera, desde 1970 se creó en el MEP, la Asesoría y Supervisión General de Planificación Familiar y Educación Sexual como órgano técnico y responsable de formular los proyectos de políticas en educación integral de la sexualidad humana. En este departamento se inscribió el Programa de Adiestramiento en Educación Sexual que, a pesar de su contenido visionario, no se tradujo en una práctica institucional y terminó archivado (Faerron, 2002).

Durante los años 1990 y 1994 el MEP elaboró y publicó, en coordinación con la Conferencia Episcopal, guías didácticas de la sexualidad humana para el III ciclo de Educación General Básica y Educación Diversificada, las cuales en la actualidad están archivadas en las bibliotecas de las escuelas y los colegios.

\footnotetext{
${ }^{5}$ Las limitaciones se extrajeron de la revisión exhaustiva de cada una de las boletas de evaluación, pues esta información no está sistematizada. Es por ello que se hizo un ejercicio de agrupación y se crearon las categorías señaladas.
} 
En el año de 1998 el país apostó a una estrategia de abordaje en el tema de la sexualidad dentro y fuera del aula con el Plan de Capacitación en Educación de la Sexualidad del Programa Amor Joven (PAJ).

EI PAJ pretendió ser la política pública que definiera el norte de la educación para la sexualidad de este país y así asegurar su continuidad en el mediano y largo plazo. Para ello, no solo intentó ser integral y novedoso, sino que se perfiló como una política con amplia participación de diferentes sectores sociales desde su misma formulación. Sin embargo, la acción de las autoridades eclesiásticas y otros grupos como el OPUS DEI impidieron su concreción y en su defecto, la política para la sexualidad vigente en la actualidad en el país dista, mucho de la que se diseñó desde el PAJ.

\subsubsection{El Programa Amor Joven}

Fue promovido desde el Despacho de la Primera Dama (administración 1998-2002) en conjunto con el Consejo Interinstitucional de Atención a la Madre Adolescente. La Secretaría Técnica se ubicó en el INAMU y aunque no fue lo programado inicialmente, se determinó que su ejecución se efectuaría desde el MEP.

Los objetivos del programa trascendieron de los enfoques hasta ahora planteados, pues gravitaron en torno a un concepto de sexualidad integral. Se formularon 6 objetivos, que en su conjunto son reveladores de la innovación que se pretendía.

EI PAJ desarrolló un esquema bajo la orientación de tres marcos: jurídico, organizativo y conceptual. En el jurídico se utilizó el marco institucional brindado por el Código de la Niñez y la Adolescencia y la Convención sobre los Derechos del Niño. Asimismo se incluyeron compromisos derivados de la Conferencia Internacional sobre Población y Desarrollo (CIPD, Cairo, 1994) y la IV Conferencia mundial de la Mujer (Beijing, 1995) que, aunque no son de acatamiento obligatorio para el gobierno, sirvieron como marco referencial.

En el marco organizativo articuló dos dimensiones, lo curricular formal impartido por personal docente, técnico y administrativo del MEP, capacitado para impartir una educación para la sexualidad integral y lo curricular informal, a través de aprendizajes informales dentro del centro educativo. En el conceptual fueron tres las dimensiones: el enfoque de género; el de derechos y el generacional. Desde el de género se advirtieron las diferencias entre mujeres y hombres, producto de la construcción cultural. Desde el de Derechos Humanos, la

${ }^{6}$ El Plan Vida Nueva es una estrategia de la actual administración estatal para el combate de la pobreza en la cual se define el trabajo en algunos cantones prioritarios. 
condición de los niños, niñas y adolescentes como sujetos de derechos y deberes. Desde el generacional, la desigualdad generada por el adultocentrismo.

Además, desde el marco conceptual se consideró no solo la condición de género y de edad, sino también la necesidad de enfrentar las condiciones de pobreza y precariedad que afectan, particularmente, a las niñas y las adolescentes.

En el plano metodológico, se pretendía la transversalización de la educación para la sexualidad en todo el currículo educativo, de manera que pudiera ser abarcada por todas las materias y no solo por aquellas que tradicionalmente la han impartido (Religión, Biología y Orientación). EI PAJ retomó las experiencias que en este campo se ejecutaron en Colombia, México y Chile. De esta manera, incluyó los Proyectos Pedagógicos en Educación de la Sexualidad y para el trabajo en las comunidades diseñó la estrategia denominada Jornadas Comunitarias de Afectividad y Sexualidad (JOCAS).

Finalmente, el Programa se concreta en 1999 e inicia con las campañas locales que incluían la distribución de materiales escritos y montaje de kioscos de información. Posteriormente se realizaron las primeras JOCAS y en forma paralela se organizaban los aspectos organizativos y operativos para iniciar el Plan de Capacitación Docente. Sin embargo, en este momento surge la reacción de la jerarquía de la Iglesia Católica.

Sacerdotes y laicos comenzaron a exteriorizar críticas y cuestionamientos en contra del PAJ. En las misas de los domingos se empezaron a distribuir volantes y se usaba el púlpito para desprestigiar el programa; en Radio María (una de las más populares en aquel momento) se hacían comentarios que no correspondían con los fundamentos del PAJ. De igual manera estos espacios se utilizaron para desacreditar a la primera dama.

El gobierno decide, entonces, conformar una Comisión mixta Iglesia - Estado. Se nombraron siete hombres, entre sacerdotes y representantes de laicos (entre quiénes estaban representantes del OPUS DEI), designados por la Conferencia Episcopal y, por el Gobierno, se designó a siete mujeres que formaban parte de la Comisión y de la Secretaría Técnica (Faerrón, 2002).

A partir de este momento el trabajo se caracterizó por largas e infructuosas reuniones; acuerdos que no se sostenían; ataques en la prensa escrita y radial en contra del PAJ y la reelaboración constante de las propuestas originales hasta que, finalmente, el programa "se perdió".

Desde un inicio la Iglesia objetó (Faerron, 2002, p. 147):

a Señalar educación de la sexualidad y no para la sexualidad. 
- Excluir del marco jurídico toda referencia a las conferencias de Cairo y Beijing por no ser vinculantes, pero además "porque la Conferencia de Beijing introduce un lenguaje ambiguo pues bajo las palabras género o salud reproductiva o derechos en el ámbito de la sexualidad se mueven conceptos equívocos, patrocinados por grupos extremos y que inducen a mucha confusión".

- La necesidad de destacar el papel de la familia en dicha educación y dejar claro el papel subsidiario del estado. Los términos adultocentrismo y construcción del sexo a través del género debían eliminarse porque contradecían el derecho y obligación de los padres a educar, criar y proteger a sus hijos conforme a sus propios valores ejerciendo la patria potestad.

- No utilizar el enfoque de género por los peligros que encierra este concepto de abrir la educación a los cinco géneros.

- El concepto "autonomía" debía eliminarse porque para la Iglesia significa "el derecho a hacer lo que quiero, con quien quiero, como quiero y cuando quiero".

- Salud reproductiva debía correr igual suerte por su posible asocio a prácticas antinatalistas y abortivas.

a Eliminar el concepto "sexualidad plena".

- Todo programa de educación para la sexualidad dirigido a niños y niñas debe ser aprobado por una junta de padres de familia de cada centro escolar.

- Era preciso hablar de "la virtud de la castidad o pureza como condición de vida realmente cristiana y como fundamento que hace posible el ejercicio de otras virtudes cristianas ya que todas son conexas e interdependientes".

u Eliminar la distinción entre adolescente embarazada y adolescente madre, pues "se es persona humana desde el momento de la concepción, por lo tanto se es madre desde el momento de dicha concepción".

Indudablemente, la oposición del Vaticano a los procesos seguidos en la elaboración de las plataformas de acción de las conferencias internacionales es un factor determinante en los anteriores planteamientos.

Hay que sumar, además, el gran poder que tiene la iglesia y que está sustentado en el artículo 75 de la Constitución Política, el cual señala la religión católica como la oficial del país.

Es decir, la estrecha relación entre la estructura confesional y la estructura política es el principal instrumento del que se sirve la Iglesia Católica para vetar cualquier política que atente contra el orden social que proclama. 
En octubre del 2000 la Iglesia rompe negociaciones y retira sus representantes de la comisión. Para informar a la comunidad nacional acerca de las razones de dicha ruptura, desplegó una amplia campaña informativa en los principales medios de comunicación. Posteriormente, la Iglesia Católica publica sus propias Guías Sexuales (Conferencia Episcopal, 2001) en cuya presentación, el Arzobispo resalta el acuerdo unánime del episcopado para romper la colaboración Estado - Iglesia en la ejecución del PAJ. Además, se agrega un artículo de la Comisión Nacional de Laicos titulado "La ideología de Género, Peligros y Alcances, Consecuencias para la Familia" en el que se resalta la diferencia entre el feminismo bueno o sea el "feminismo de la equidad" (el que desea una sana y verdadera reivindicación de la mujer) y el feminismo malo, es decir "el feminismo de género" (basada en el enfrentamiento de la mujer contra el hombre, logrando con esto la desintegración familiar).

\subsubsection{Políticas de Educación Integral de la Expresión de la Sexualidad Humana}

En el período de negociaciones de la Iglesia y el Estado, se elaboraron varias propuestas que trataban de incorporar las demandas de la primera. No obstante, se dio el rompimiento señalado, y el MEP as umió la conducción del proceso.

Finalmente, en junio 2001 se publican las Políticas de Educación Integral de la Expresión de la Sexualidad Humana, las cuales inician con una cita del cardenal Alfonso Trujillo López ${ }^{7}$ :

La sexualidad humana es un bien: parte del don que Dios vio que "era muy bueno" cuando creo la persona humana a su imagen y semejanza, y "hombre y mujer los creo". En cuanto a modalidad de relacionarse y abrirse a los otros, la sexualidad tiene como fin intrínseco el amor, más precisamente el amor como donación y acogida, como dar y recibir" (MEP, 2001a, p. 4).

Iniciar una política educativa con una cita religiosa denota el interés del Estado de no confrontar a la Iglesia Católica. Ello es aun más evidente si se observa la inclusión de algunos de los aspectos que habían sido objeto de la controversia inicial con la iglesia. Así por ejemplo, entre otros, se estableció que la familia tiene el derecho y el deber de la educación de la expresión de la sexualidad humana y la escuela tiene una acción subsidiaria; se eliminó para la sexualidad y se utiliza de la sexualidad y no aparecen los conceptos de autonomía, salud reproductivay sexualidad plena.

\footnotetext{
${ }^{7}$ Cita extraída del libro "Sexualidad Humana: Verdad y Significado" del Cardenal Alfonso López, quien según Faerron (2002.) es identificado con la línea conservadora de la Iglesia Católica. 


\subsubsection{Departamento de Educación Integral de la Sexualidad Humana}

En agosto 2000 y paralelo a las controversias suscitadas con la iglesia, surge en el MEP el Departamento de Educación Integral de la Sexualidad Humana, cuyo antecedente era la oficina de Proyectos Especiales ubicada en el Centro Nacional de Didáctica (CENADI). En julio 2001 se estructura como departamento responsable de la educación integral de la sexualidad humana en la población estudiantil de todos los niveles, ciclos y modalidades ajustándose, para ello, con los postulados y definiciones aprobados por el Consejo Superior de Educación en las Políticas de Educación Integral de la Expresión de la Sexualidad Humana.

A pesar de que ya existía para ese entonces, el departamento no participó en la comisión del MEP que elaboró las Políticas de Educación Integral de la Expresión de la Sexualidad Humana.

Existen serios indicios que sugieren una función marginal del departamento pues a la fecha no se ha elaborado un plan nacional que aglutine acciones concretas y responsabilidades específicas por parte de las diferentes instancias de la institución, que hagan efectivo el cumplimiento de las mencionadas políticas (Defensoría de los Habitantes, 2003).

(....) Llama la atención a esta Defensoría que al revisar y analizar los informes que remite el Ministerio de Educación Pública (Despacho de Ministra y Despacho de Vice Ministro Académico respectivamente) pareciera que, en el nivel interno de esa institución, no existe coordinación y cooperación con el Departamento de Educación Integral de la Sexualidad Humana, así como tampoco un liderazgo de este Departamento en la toma de decisiones y ejecución de acciones en esta materia (Defensoría de los Habitantes, 2003, p. 22).

La falta de sostenibilidad es observable en los programas ejecutados pues a pesar de que el departamento retomó los centros educativos de las 5 regiones con las que trabajó el Proyecto de Sexualidad de la Oficina de Población (CENADI) el trabajo que este proyecto inició no se pudo continuar debido "a que la intervención no se sistematizó y a que muchos de los proyectos no eran tales sino actividades sueltas, por ejemplo una charla sobre abuso sexual (Araya, 2003c).

\subsection{Oficina de Equidad de Género}

Por medio de un decreto ejecutivo firmado en febrero del año 2000 se crea esta oficina, a través de la consolidación y nuevas atribuciones de la OMM/MEP. Con este decreto se 
estableció el compromiso del MEP para la promoción de la equidad de género en su quehacer institucional. Asimismo, en esta fecha, se firmó un Convenio de Cooperación entre el MEP y el INAMU.

En cumplimiento de lo estipulado en ambos instrumentos, el MEP integró una Comisión de alto nivel técnico y político, conformada por representantes de dependencias claves, con el fin de que se responsabilizaran de las acciones y decisiones que al respecto se tomaran. Con el respaldo técnico del INAMU se diseñó el Plan de Acción "El Sistema Educativo hacia una Cultura de Equidad de Género" (MEP; s.f).

El Plan contiene 21 acciones de carácter estratégico tendientes a favorecer el acceso igualitario de las mujeres a los servicios que ofrece el MEP, así como a orientar estos servicios desde la perspectiva de las necesidades y los intereses de la población femenina. Incluye también acciones dirigidas a modificar el ambiente laboral y la cultura organizacional del Ministerio.

Según la estrategia de intervención institucional diseñada por el INAMU, las entidades de gobierno debían asumir la ejecución de las acciones del Plan de Acción Estratégico como parte de las funciones propias de sus dependencias, lo que implicaba su incorporación dentro de los planes anuales operativos PAOs. Con esto se pretendía asegurar la adecuada asignación presupuestaria y su inclusión dentro de los procesos de evaluación institucional. Aspecto que no ocurrió así.

El decreto establece un informe de rendición de cuentas y desde el 2002 hasta la actualidad, se evidencian diferencias sustantivas respecto al ritmo de avance en cada una de las acciones. Algunos de los factores que determinan la diferencia de avance están ligados a la disponibilidad de recursos económicos, las prioridades en la agenda de trabajo del ministerio y el compromiso asumido con la equidad de género por las autoridades políticas (Araya, 2003d).

Con recursos económicos del INAMU se elaboraron unos módulos para la inclusión del enfoque de género en los programas de estudio de las materias básicas en primaria y en secundaria. No obstante, solo hay 100 ejemplares y el INAMU está buscando recursos externos que permitan su tiraje y su uso en 2004.

Por último, valga destacar algunos elementos en cuanto a la Oficina de Equidad de Género y el Plan "El Sistema Educativo hacia una Cultura de Equidad de Género".

口 Cuando se creó, la Oficina estuvo adscrita al despacho del Viceministro Académico.

Contaba con un espacio propio en el edificio que alberga los despachos de la ministra y de los viceministros. Desde un inicio y hasta la actualidad no ha tenido Volumen 3, Número 2, Año 2003 
presupuesto, sino que depende de la asignación de montos para actividades específicas (afiches, conmemoraciones, viáticos, etc.). Por ello es desde el INAMU donde se apoya el material didáctico y pago de especialistas, con el agravante de que los recursos de esta institución también son restringidos.

- Solo cuenta con una funcionaria a quien corresponde la realización de las actividades de distinta naturaleza (coordinación, secretariales, convocatorias, etc.).

- A partir de julio del 2002 la Oficina es trasladada a otro edificio del MEP, lo que generó un sentimiento de exclusión en la coordinadora. Las autoridades ministeriales deciden que la Oficina no dependa más del despacho del Viceministro, sino del departamento de Educación para la Sexualidad. Según la coordinadora, este movimiento no solo invisibilizó sino que también inmovilizó las acciones pues desde la fecha del traslado hasta febrero del 2003, la Oficina se sumió en este departamento. Es en esta última fecha en la que se decide que la Oficina debe reportar a la División Curricular. No obstante, este movimiento no significó la asignación de presupuesto.

- Durante el año 2000 y parte del 2001, la Oficina estuvo abocada a la coordinación para la elaboración del Plan de Acción. Esta labor no resultó lo fluida que se esperaba pues a pesar de que el decreto establecía que la comisión redactora sería de alto nivel — de forma que la conformarían las jefaturas de as distintas divisiones del MEP- ello no fue así. Las jefaturas, "con excepción de unas pocas, dejaron de participar y delegaron su participación en un personal sin poder de decisión, lo que generó que la comisión no se consolidara y se atrasara la toma de decisiones" (Araya, 2003d). Esta comisión se extinguió en el 2002 y en la actualidad, la Oficina coordina en forma independiente y atomizada con los departamentos del MEP que asumieron acciones particulares.

- La coordinadora participa como representante del MEP en diversas comisiones, generando multiplicidad de acciones, algunas sin ninguna vinculación entre ellas.

\subsection{Inserción de las mujeres en especialidades técnicas-profesionales}

La preocupación por la inserción de las mujeres en carreras competitivas se inició en el período 1996-1998, por medio de la ejecución de un proyecto financiado por la Unión Europea y ejecutado por el INAMU y el Departamento de Educación Técnica Profesional (DETP) del MEP. 
La experiencia se desarrolló en siete colegios técnicos y tuvo como fin primordial instar a las estudiantes a optar por especialidades no tradicionales, enfatizando en Electromecánica e Informática.

Los resultados del proyecto no se sistematizaron y se desconocen sus alcances y logros. No obstante, es considerada como una experiencia valiosa, pues se logró revisar los programas de estudio para eliminar los sesgos de género en algunas de estas carreras y se desarrollaron una serie de panfletos y de videos (Araya, 2003e). Pese a ello, una vez que finalizó el financiamiento de la Unión Europea, no se dio ningún seguimiento por no contar el MEP con el respectivo presupuesto.

La formación técnica de las mujeres es retomada, posteriormente, en el Plan de Acción "El Sistema Educativo hacia una Cultura de Equidad de Género", específicamente en el diseño y la aplicación de la acción denominada "Inserción paritaria de mujeres y hombres en especialidades técnicas diversas y competitivas". No obstante, en esta ocasión tampoco se retomaron las experiencias derivadas del proyecto financiado por la Unión Europea.

La acción "Inserción paritaria de mujeres y hombres en especialidades técnicas diversas y competitivas" se llevó a cabo en 5 colegios y se desarrolló por medio de cuatro componentes con sus respectivos objetivos y actividades: a) elaboración de un diagnóstico, b) sensibilización y capacitación; c) apoyo a la toma de decisiones y elección vocacional y, d) plan de incentivos.

Algunos de los logros de este proyecto son bastante significativos en tanto se visibilizó que la paridad en la matrícula no ha generado cambios en la elección vocacional. La fuerte concentración de la matrícula femenina en las áreas de comercio y servicios así lo hizo evidente. (Ibarra, s.f.).

El proyecto ha estado paralizado por falta de contenido presupuestario y porque el DETP se abocó a una revisión de las carreras técnicas. En la actualidad se retomó, pero no se tiene programado trabajar en los mismos centros educativos debido a la falta de interés del personal docente (Araya, 2003b).

A pesar de que se cuenta con la voluntad política de las más altas autoridades y de los mandos medios para transversar el género, cuando el lineamiento llega al nivel ejecutor el compromiso pierde fuerza, lo cual dificulta la ejecución de las acciones tendientes a lograr la equidad en la prestación de los servicios. En otras ocasiones, aunque existe voluntad y compromiso, el personal clave no logra traducir las políticas o acciones utilizando la visión de género; a ello se adiciona las dificultades que enfrentan 
los/as funcionarios/as para hacer análisis diferencial de género y para convertir los diagnósticos en propuestas concretas para las mujeres (Ibarra, s.f. p. 39).

Sin establecer una estrategia para la superación de las limitaciones identificadas, $d$ Plan Educativo 2002-2006 (MEP, 2002b), señala una única acción con relación a la inclusión del género y es, precisamente, la referida a la equidad de género en la formación técnica. En la misma se define como meta aumentar en $20 \%$ la matrícula de mujeres registrada en Electromecánica, Electrotecnia, Electrónica General, Electrónica Industrial, Electrónica Reparación de Equipos de Cómputo, Mecánica de Precisión y Microelectrónica.

Con lo anterior se desconoce o ignora que la paridad numérica es una condición necesaria, pero no suficiente. Efectivamente, los esfuerzos que en esta dirección se realicen serán infructuosos si no se aborda en forma paralela el cambio de actitudes de las empresas contratantes dada la evidencia (Guzmán, 1999) de que el repunte en la matrícula y la opción por las carreras competitivas no se han traducido en mejores condiciones de empleo ni salariales para las mujeres.

\section{Síntesis}

a No se puede desmerecer la inclusión del género en la agenda institucional del MEP pero esto ha sido nominal en la medida que la no asignación de presupuesto, limita su ejecución.

a Desde 1981 se ha señalado que el presupuesto es de vital importancia para las acciones operativas que se derivan de las reformas. De igual manera, desde esta fecha, se resalta a necesidad de que el magisterio y las autoridades educativas participen en las reformas desde su génesis y no solo en su ejecución lo que permitiría una mayor comprensión de sus alcances y de su operacionalización. Ambas recomendaciones, hasta la fecha, continúan sin ser atendidas.

- Los planes, proyectos y planes para la equidad de género se efectúan en forma atomizada y sin ningún encadenamiento entre ellos por lo que carecen de un impacto significativo.

a Para que las políticas tengan los efectos programados deben sustentarse en una visión de mediano o largo plazo. La visión cortoplacista que es intrínseca a las políticas educativas afecta en forma especial las políticas de género en la medida que ellas implican cambios culturales y cualquier cambio cultural exige procesos sostenidos. 
a La Oficina de Equidad de Género no tiene acción vinculante con otras oficinas y se llena de una multiplicidad de tareas en las que se diluyen los objetivos por los cuales fue creada.

- El MEP es una institución en la que se mantienen estructuras desiguales y rígidas entre las altas jerarquías, personal administrativo, docentes y alumnas/os y ello produce que las acciones marchen en forma lenta o bien solo queden en el plano de la formulación.

- El surgimiento del PAJ en un estamento de poder como el de la Primera Dama trae como consecuencia su asociación a un color político. Quizás — pues para saberlo con certeza se requiere de una investigación- este fue el motivo por el que las universidades y las ONG no se pronunciaron a favor del PAJ. "Mientras se recibían 500 telegramas de madres y padres y docentes solicitando al MEP que cercenara al $P A J$, las universidades e Institutos del Niño de este país guardaban silencio. (Araya, 2003c).

- Hay una tendencia a abordar los temas de equidad de género solo en el nivel de primaria. Esto obedece no solo a factores endógenos, sino también a las prioridades definidas por los organismos internacionales.

- Algunos programas son reiterativos, pues aunque cambian los sustentos conceptuales, las acciones terminan siendo réplicas de otras ya ejecutadas sin que exista un proceso de reflexión acerca de las limitantes que obstaculizaron la ejecución de las segundas (material didáctico, oficina de la mujer, inserción en carreras técnicas, por ejemplo).

- La exigencia de trabajar los 200 días es una de las grandes limitantes para la capacitación docente pero, además, la constante rotación de personal docente, incide también en la poca sostenibilidad de los programas.

\section{Conclusiones}

Cualquier política pública que intenta ser eco de problemáticas sociales controvertidas y no legitimadas por la tradición escolar, siempre va a enfrentar distintos avatares. No obstante, sin ánimo de caer en conclusiones autoreferentes, lo cierto es que las políticas de equidad de género encuentran resistencias que devienen de las movilizaciones personales que generan. Dichas resistencias se evidencian desde la propia formulación y posterior ubicación de los programas en la estructura administrativa y espacial, hasta la no asignación de presupuestos institucionales que inhiben o impiden su impacto. 
Innegable es el avance en materia jurídica y la visibilización de la temática en la agenda pública nacional, sin embargo, ello no es suficiente. Se requiere que los cambios en el lenguaje no solo sean nominales sino también en el orden simbólico, que la modificación de los materiales didácticos sea acompañada por un proceso permanente de reflexión docente y lo más importante, aunar esfuerzos para que el género deje de ser satanizado por las distorsiones que han acompañado en forma paralela su desarrollo conceptual.

Abordar - y creer en - la equidad de género significa, en primer término, la supresión de las dualidades sobre las cuales descansan las desigualdades sociales. Es decir, significa comprender que estas dualidades son producto de la construcción cultural y por tanto se incorporan en la cotidianeidad de las personas como "naturales" (norma - anormal, buena mala, cuerpo- mente; femenino - masculino) por lo que es necesario abordarlas hasta lograr que el conjunto de normas sociales se transforme. Solo de esta manera será posible fusionar los valores, las actitudes y las prácticas históricamente atribuibles, por un lado a las mujeres $y$, por el otro, a los hombres. Cuando esto ocurra habremos transformado los géneros hasta llevarlo a su desaparición.

La complejidad y verticalidad de las instituciones del Estado y, en particular, las que se encargan de la educación, entrañan en su interior grandes y poderosos conflictos de intereses provenientes de las dosis de poder ostentadas por cada uno de los departamentos o instancias que las conforman. Las representaciones sociales prevalecientes acerca de la sexualidad, de la igualdad, de lo femenino y lo masculino, son las orientadoras de as conductas de las personas y por ende se reflejarán en las políticas que formulen y en la forma en que conduzcan los procesos educativos. $Y$ en los microespacios, como lo son las aulas educativas, para nadie es un secreto el gran poder del cuerpo docente. De ahí, que cualquier política o programa puede ser resignificada según sean las representaciones de los y las docentes.

Los anteriores aspectos se conjugan y sugieren que es más viable un proyecto de equidad de género planteado desde las bases docentes que desde las cúpulas educativas. Es decir, la complejidad institucional de los espacios estatales de educación limita la sostenibilidad requerida por los proyectos y programas de equidad de género.

Apostar, por tanto, a la formación docente inicial como a la formación continua es una alternativa para enfrentar los avatares institucionales. En este sentido, la coordinación con las oficinas de la mujer de los gremios docentes constituye un buen inicio para llevar a cabo la capacitación del personal de servicio. 
Sin embargo, es la formación inicial a la que mayor apuesto por considerar que es un espacio propicio para la creación y la modificación. Para lograr el cambio en y desde la educación es preciso, por tanto, que en los procesos de formación docente se haga un reconocimiento del valor de las desigualdades sociales procedentes de la construcción cultural de los géneros. No es el currículo común, ni la metodología homogénea, ni la paridad numérica las que garantizarán la igualdad de oportunidades para las mujeres y los hombres. Lo será, más bien, la atención a las diferencias individuales, mediante una metodología y un currículo lo suficientemente flexibles y diversificados como para garantizar el acceso de las mujeres y los hombres a un conjunto de saberes, habilidades y destrezas que les permiten estar en mejores y más iguales condiciones de vida.

Cierto es que en los programas iniciales no encontramos ningún esfuerzo sistemático de formación o introducción de una perspectiva de equidad de género y, es probable, que esto sea producto de las tradiciones de formación docente prevalecientes en las facultades de educación. No obstante, considero que el reto actual en educación es penetrar en ellos y convertirlos en la plataforma en donde inicie la creación de condiciones para la equidad de género. 


\section{Referencias}

Araya, Sandra (2001). La equidad de género desde la representación de las formadoras y los formadores del profesorado de enseñanza secundaria. San Pedro, Costa Rica. Tesis para optar al grado de Doctor en Educación: Universidad de Costa Rica.

Araya, Sandra (2001). La equidad de género en la educación. Revista de Estudios de Género La Ventana. Volumen II, № 13 . Universidad de Guadalajara, México.

Araya, Sandra (2003). Comunicación personal. 27 de junio 2003. Instituto Nacional de las Mujeres.

Araya, Sandra (2003). Comunicación personal. 28 de marzo, 2003. Ministerio de Educación Pública: Departamento de Educación Integral de la Sexualidad Humana

Araya, Sandra (2003). Comunicación personal. 17 de marzo, 2003. Ministerio de Educación Pública: Oficina de Equidad de Género.

Araya, Sandra (2003). Comunicación personal. 28 de marzo del 2003. Ministerio de Educación Pública: Departamento de Educación Técnica.

Araya, Sandra (2003). Un matrimonio conveniente: el género y la educación. Revista Educación. Vol. 27, № 2. Instituto de Investigaciones para el Mejoramiento de la Educación Costarricense, Universidad de Costa Rica.

Araya, Sandra (s.f.). Las representaciones sociales: Ejes teóricos para su discusión. Cuadernos de FLACSO. En prensa.

Arriagada Irma (1998). Políticas públicas y de género. Una relación difícil. Revista Paraguaya de Sociología. Año 35, № 101.

Carr, Wilfred. Una teoría para la educación. Hacia una investigación educativa crítica. Madrid, España: Morata, 1996.

Centro Nacional para el Desarrollo de la Mujer y la Familia (1996). Plan para la Igualdad de Oportunidades entre Mujeres y Hombres. San José, Costa Rica: El Centro.

Centro Nacional para el Desarrollo de la Mujer y la Familia (1998). Informe de labores 19941998. San José, Costa Rica: El Centro.

Centro Nacional para el Desarrollo de la Mujer y la Familia. (1997a). Descubriendo mi socialización de género. Primer módulo. San José, Costa Rica: El Centro.

Centro Nacional para el Desarrollo de la Mujer y la Familia. (1997b). Armando el rompecabezas. Segundo módulo. San José, Costa Rica: El Centro.

Centro Nacional para el Desarrollo de la Mujer y la Familia (1997c). Haciendo camino al andar. Tercer módulo. San José, Costa Rica: El Centro.

Conferencia Episcopal de Costa Rica (2001). Educación en la Sexualidad, Guía para Padres de Familia y Maestros. San José, Costa Rica. 
Chavarría, Soledad, Paniagua María Eugenia, Quesada Sheila (1998). La Política Educativa hacia el Siglo XXI: propuestas y realizaciones. Tomo I. San José, Costa Rica: Ministerio de Educación Pública.

Defensoría de los Habitantes (2003). Denuncia Daria Suárez. Informe Final con recomendaciones. Expediente №13256-26-2002. San José, Costa Rica.

Despacho de la Primera Dama de la República (1999). Programas Amor Joven y Construyendo Oportunidades. San José, Costa Rica: Despacho de la Primera Dama de la República.

Faerron, Ana Lucía (2002). La educación para la sexualidad en el contexto de la sociedad costarricense: Análisis del proceso de diseño y gestión del Programa Amor Joven (1998-2001). Costa Rica. Tesis pata optar al grado de Magíster Scientiae en Estudios de la Mujer: Universidad de Costa Rica.

Fernández, Emma (2001). Sub-proyecto Construcción de una cultura equitativa de género en la escuela líder. En Sistematización del proyecto de apoyo al SIMED: Un aporte con significancia cualitativa a la educación costarricense de I y II ciclos. Los subproyectos: una visión holística y tranversal para enriquecer el currículo. San José, Costa Rica: EDITORAMA.

González, M. (1987). Sexismo y educación escolar en Costa Rica. Madrid, España, Universidad Autónoma de Madrid. Tesis doctoral.

González, M. (1990). El sexismo en la educación: la discriminación cotidiana. Ciudad Universitaria Rodrigo Facio: Editorial de la Universidad de Costa Rica.

Guzmán, Laura (1999). Informe de Consultoría. Sistematización y lineamientos para una propuesta teórico-metodológica de trabajo con mujeres adolescentes y jóvenes con perspectiva de género. San José, Costa Rica: Programa Mujeres Adolescentes, Unión Europea ALA 92/46.

Ibarra Marta (s.f). El género como eje transversal en la educación técnica media. San José, Costa Rica. Documento preparado por el Instituto Nacional de las Mujeres para el IV Congreso Iberoamericano de Ciencia, Tecnología y Género.

Ibarra, M. (1997). Metodología para eliminar estereotipos sexuales en la educación y comunicación social. Panamá: UNICEF.

Instituto Nacional de las Mujeres (2002). Agenda y Metas Institucionales: 2002-2006. Igualdad, Equidad de Género y Derechos Humanos de las Mujeres. San José, Costa Rica. Mimeo.

Michel, A. (1987). Fuera moldes. Hacia una superación del sexismo en los libros infantiles y escolares. Barcelona, España: Editorial Los Dones.

Ministerio de Educación Pública (1981). Informe nacional sobre experiencias de implementación de reformas educativas llevadas cabo en Costa Rica. Costa Rica: MEP. 
Ministerio de Educación Pública. (1993). Sistema educativo nacional de Costa Rica. Costa Rica. Ministerio de Educación Pública, Organización de los Estados Iberoamericanos.

Ministerio de Educación Pública (1998). El Qué y el Cómo de la Proyección de las Escuelas Líderes. San José, Costa Rica: SIMED - UNESCO/Países Bajos.

Ministerio de Educación Pública. (2001). Políticas de Educación Integral de la Expresión de la Sexualidad Humana. San José, Costa Rica: MEP.

Ministerio de Educación Pública. (2002). Taller de Seguimiento, asesoría y sistematización del Subproyecto Construcción de una cultura de equitativa de género en las escuelas líderes. Material de trabajo.

Ministerio de Educación Pública. (2002). Plan Educativo. Período 2002- 2006. San José, Costa Rica: MEP.

Ministerio de Educación Pública (s.f). El sistema Educativo Nacional. Hacia una cultura de Equidad de Género. Plan de Acción Estratégico 2000-2002. San José Costa Rica: MEP.

Montes de Oca, M., Rosales, A (1994). Guía para eliminar el sexismo en los cuentos. San José, Costa Rica: Centro Nacional para el Desarrollo de la Mujer y la Familia.

Montes de Oca, M., Rosales, A. (1993). El sexismo y los cuentos infantiles de autores y autoras costarricenses para niños y niñas de 3 a 6 años. San Pedro, Costa Rica. Universidad de Costa Rica (tesis de licenciatura).

Paniagua, Ma. Eugenia (2002). Política educativa. El caso Costa Rica. San José, Costa Rica. Ponencia presentada en el Simposio de Educación.

Paredes, R. (1994). Metodología para eliminar estereotipos sexuales en los materiales educativos. Para una educación sin disparidades de género. Guatemala: Ediciones UNICEF.

Paredes, R., Sánchez B., Caldera R. (1994). El sexismo en los textos escolares en Centroamérica. Propuesta de una estrategia para su eliminación. Guatemala: Ediciones UNICEF.

Ramellini, Teresita (2000). La situación de la violencia de género contra las mujeres en Costa Rica. San José, Costa Rica: Programa de las Naciones Unidas para el Desarrollo.

Sánchez, Zaida. (2002). Reformas Educativas en Costa Rica. San José, Costa Rica: Ministerio de Educación Pública.

Schneckenberg, Marisa. (2003). La implementación del PROEM (Programa Expansión, Mejora e Innovación en la Enseñanza Media) como política educativa en la práctica de la gestión escolar. Argentina: IIPE-UNESCO. 
Stromquist, Nelly. (2003). Consideraciones teóricas y programáticas sobre las políticas de equidad en América Latina. Una introducción al tema. Ponencia presentada en el 51 Congreso de Americanistas, Chile.

UNESCO. (1992). Recomendaciones para un uso no sexista del lenguaje. Folleto

UNICEF (1990). Mujer y sociedad. Educación. (Cartillas de trabajo).

UNICEF/UNIFEM. (1990). Manual de recomendaciones para la eliminación de estereotipos sexuales en los textos escolares y en los materiales educativos en general. Guatemala: UNICEF.

UNICEF/UNIFEM. (1991). Análisis de roles y estereotipos sexuales en textos escolares en Costa Rica. San José, Costa Rica: UNICEF/UNIFEM. 